\section{Visión Electrónica Más que un estado sólido \\ https://doi.org/10.14483/issn.2248-4728}

UNIVERSIDAD DISTRITAL

FRANCISCO JOSÉ DE CALDAS

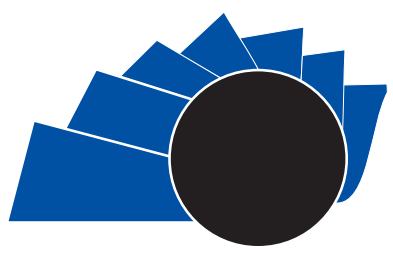

VISIÓN ELECTRONICA

Visión Investigadora

\title{
Microturbina operada con biogás generado por estiércol bovino
}

\author{
Microturbine operating with biogas generated by cattle manure \\ Angel David Rodríguez Vargas ${ }^{1}$, Laura Andrea Morales García ${ }^{2}$, Herbert Enrique Rojas Cubides ${ }^{3}$
}

\section{INFORMACIÓN DEL ARTICULO}

Historia del articulo

Enviado: 29/11/2017

Recibido: 11/03/2018

Aceptado: 05/07/2018

\section{RESUMEN}

El artículo presenta los resultados del análisis del comportamiento de un sistema de generación distribuida compuesto por una microturbina operada con biogás, variando una carga característica de una zona no interconectada (ZNI). Para ello, se identifica una ZNI con alto potencial para el aprovechamiento eléctrico del biogás usando datos estadísticos de producción pecuaria y cobertura eléctrica; luego se estima la producción de biogás y su composición aplicando el modelo de digestión anaerobia (ADM1)

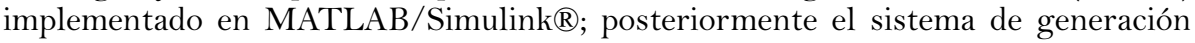
distribuida es simulado usando el mismo entorno de programación. Se obtuvo que con el $1 \%$ del estiércol producido por la industria bovina de San Vicente del Caguán (66203,6 kg/día) sería posible generar 78868 kg de metano en 100 días; con esta cantidad de biocombustible se podría abastecer la demanda eléctrica promedio $(29,97$ $\mathrm{kW}$ ) de la localidad San Juan de Caquetania (Colombia) durante 444 días usando una microturbina de $65 \mathrm{~kW}$.
Keywords:

ADM1

Biogas

Cattle manure

Microturbine

\begin{abstract}
:
This article presents the results of analyzing the behavior of a distributed generation system composed by a microturbine operated with biogas, varying the characteristic load of a non-interconnected zone (ZNI). For it, identifies a ZNI with high potential for the electric use of the biogas using statistical data of livestock production and electrical coverage; then, the biogas production and its composition are estimate applying the

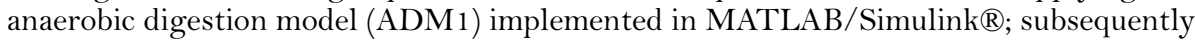
the distributed generation system is simulated using the same programming environment. It achieved that with $1 \%$ of the manure produced by the cattle industry of San Vicente del Caguán $(66203,6 \mathrm{~kg} /$ day) it would be possible to generate $78868 \mathrm{~kg}$ of methane in 100 days. With this amount of biofuel, the average electrical demand $(29,97 \mathrm{~kW})$ of the town of San Juan de Caquetania (Colombia) could be supplied for 444 days using a $65 \mathrm{~kW}$ microturbine.
\end{abstract}

\footnotetext{
1 Ingeniero Eléctrico, Universidad Distrital Francisco José de Caldas, Colombia. Grupo de Investigación en Compatibilidad e Interferencia Electromagnética, Universidad Distrital Francisco José de Caldas. Bogotá, Colombia. Lugar de trabajo: Ingeniero de Proyectos, EPC Ingeniería. Correo electrónico: andarodriguezv@gmail.com. ORCID: https://orcid.org/0000-0003-1704-9911.

2 Ingeniera Eléctrica, Universidad Distrital Francisco José de Caldas, Colombia. Grupo de Investigación en Compatibilidad e Interferencia Electromagnética, Universidad Distrital Francisco José de Caldas. Bogotá, Colombia. Lugar de trabajo: Ingeniera de diseño, Inmel Ingeniería. Correo electrónico: laamoralesg@gmail.com. ORCID:

https://orcid.org/O000-0001-6324-6452.

3 Ingeniero Electricista, MSc. y Candidato a Doctor en Ingeniería Eléctrica, Universidad Nacional de Colombia, Profesor Asociado, Proyecto Curricular de Ingeniería Eléctrica, Grupo de Investigación en Compatibilidad e Interferencia Electromagnética, Universidad Distrital Francisco José de Caldas, Bogotá, Colombia. Correo electrónico: herojasc@udistrital.edu.co.oRCID: http://orcid.org/0000-0003-1253-6964.

Citar este artículo como: A. D. Rodríguez-Vargas, L. A. Morales-García y H. E. Rojas-Cubides, "Microturbina operada con biogás generado por estiércol bovino”, Visión electrónica, algo más que un estado sólido, vol. 1, no. 1, Edición especial, enero-junio 2018. DOI revista: https://doi.org/10.14483/issn.2248-4728.
} 


\section{Introducción}

En Colombia, la imposibilidad de llevar energía eléctrica a algunas zonas remotas y rurales ha hecho que dichos lugares presenten un rezago social, económico y tecnológico, lo cual es alarmante si se tiene en cuenta que las zonas no interconectadas $(\mathrm{ZNI})$ representan el $52 \%$ de la extensión del territorio nacional, [1]. Para solventar el déficit eléctrico de las ZNI se recurrió a la generación distribuida, la cual en el contexto colombiano se basa en motores diésel que impulsan pequeños generadores eléctricos. Estos generadores muchas veces son insuficientes para dar 24 horas de cobertura eléctrica, [2]. Adicionalmente, los motores diésel producen emisiones y elevados costos de mantenimiento y operación debido al precio del combustible, lo que crea la necesidad de usar otras fuentes de generación de energía que aprovechen los recursos renovables disponibles en las ZNI, [3] .

En este contexto surge como alternativa el biogás, el cual puede ser usado como fuente de energía renovable para la producción de energía térmica, mecánica y eléctrica, [4]. Este biocombustible es producido a partir de la descomposición controlada de residuos orgánicos en un proceso bioquímico y fisicoquímico llamado digestión anaerobia (DA), [5]. Con este proceso se logran beneficios ambientales, sociales y económicos, lo que ha llevado a varios países a formular proyectos orientados a masificar su uso mediante esquemas de energización rural que fortalezcan las actividades productivas de una región, [3]. No obstante, este biocombustible no ha sido ampliamente utilizado en el país debido a la falta de conocimiento sobre el proceso de DA y a los pocos incentivos financieros para este tipo de proyectos, [6] .

Como la DA es un proceso complejo y no lineal que depende de muchos aspectos como la temperatura, el $\mathrm{pH}$, la relación carbono nitrógeno y la composición del residuo de entrada, se hace difícil estimar su comportamiento [4],[7]. Por lo anterior, una de las herramientas más usadas para representar las etapas de la DA y estimar la producción de biogás son los modelos matemáticos. Es así que a lo largo de los años se han desarrollado diferentes modelos que representan los principales aspectos biológicos y bioquímicos de la DA, permitiendo mejorar la comprensión del sistema y la predicción del proceso bajo distintas condiciones de operación [8]. Uno de los modelos más utilizados y referenciados es el Modelo de Digestión Anaerobia $\mathrm{N}^{\circ} 1$ (ADM1), que permite describir el proceso de la DA de manera general para diferentes tipos de reactores, parámetros operacionales y características en el sustrato de entrada, [9]. Además, el ADM1 es capaz de realizar simulaciones dinámicas de la DA y optimizar diseños, [8].

Por otro lado, la conversión de biogás a electricidad se puede lograr utilizando diferentes tecnologías de conversión como el motor de combustión interna (MCI), la celda de combustible (CC) y la turbina de gas (TG), [4] . En la mayoría de los casos, se usa un MCI acoplado a un generador eléctrico. Aunque los MCI pueden lograr eficiencias de hasta $40 \%$ (cuando tienen sistemas de recuperación de calor), estos presentan mayores emisiones de gases de efecto invernadero y costos de mantenimiento, [4].

Por su parte, las CC son las que poseen mejor eficiencia de conversión (hasta 70\%), pero aún se encuentran limitadas a aplicaciones experimentales debido a su elevado costo de inversión y su corta vida útil, [10]. En contraste, las turbinas y microturbinas son las que presentan menores costos de inversión y relación costo/kWh. Adicionalmente, presentan gran flexibilidad para usar diferentes tipos de combustible, desde gases de bajo poder calorífico como el biogás hasta combustibles líquidos, [4].

Teniendo en cuenta estas consideraciones, esta investigación analiza el comportamiento de una microturbina conectada a red aislada que es representada como una carga resistiva, la cual se dimensiona de acuerdo a la demanda insatisfecha de una ZNI. La microturbina es alimentada con biogás producido por la DA del estiércol bovino. Este recurso se definió considerando que la mayoría de las ZNI basan su economía en el sector pecuario, principalmente en la ganadería bovina.

El trabajo, que estructura al mismo tiempo el documento, se desarrolla en dos etapas: primero se selecciona como caso de estudio una ZNI con buen potencial para el aprovechamiento eléctrico del biogás y usando el ADM1 se calcula la posible producción de biogás a partir del estiércol bovino disponible. Posteriormente, se estima el comportamiento del sistema de conversión biogás-electricidad utilizando el modelo de Rowen para simular la TG [11] y los bloques disponibles en la biblioteca de Simulink ${ }^{\circledR}$ para el generador sincrónico y las etapas de acondicionamiento de las señales de potencia (Conversor AC-DC-AC).

\section{Modelo de digestión anaerobia $\mathrm{N}^{\circ} 1(\mathrm{ADM} 1)$}

El ADM1 está basado en procesos bioquímicos realizados de manera secuencial y paralela por varios grupos microbianos en reactores sellados, denominados 
biodigestores.

En los cuales la materia orgánica es descompuesta en bioabono y biogás [9], [12]. Un esquema simplificado del modelo es mostrado en la Figura 1, d $\mathrm{o}$ n d e . $q_{\text {out }}$ es el afluente y $q_{\text {gas }}$ es el flujo de biogas producido.

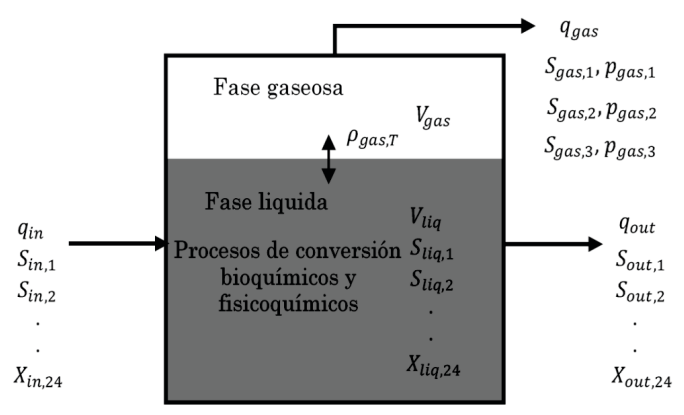

Figura 1 . Esquema del ADM1 en un biodigestor tipo de un solo tanque [9] .

En su estructura, el ADM1 se divide en una fase liquida y otra gaseosa las cuales se relacionan mediante tasas de transferencia de masa liquido-gas ( $\rho_{\text {gas,T }}$ ). La fase liquida reúne las concentraciones de los componentes del residuo de entrada y las que se encuentran dentro del reactor, mientras que la fase gaseosa agrupa los gases producidos por la descomposición de la biomasa al interior del biodigestor.

Adicionalmente, el ADM1 se basa en dos tipos de reacciones, las bioquímicas y las fisicoquímicas. Las primeras describen tres pasos biológicos generales (acidogénesis, acetogénesis y metanogénesis) e incluyen un paso de desintegración extracelular y uno de hidrólisis extracelular. Entre tanto, las funciones fisicoquímicas describen fenómenos que no son mediados por los microorganismos pero que afectan directa o indirectamente el crecimiento de las poblaciones bacterianas ( $\mathrm{pH}$, equilibrio acido-base y las concentraciones de gases), [9].

Con respecto a la matemática del modelo, este tiene 29 variables de estado con un comportamiento dinámico, las cuales tienen un balance de masa representado por la ecuación (1). Las variables se dividen en 26 para la fase líquida y 3 para la fase gaseosa (concentraciones de metano, dióxido de carbono e hidrógeno),

$\frac{d m_{x}}{d t}=\dot{m}_{x, \text { in }}-m_{x, \text { out }}+\dot{r}$

En la ecuación (1) $\mathrm{m}_{\mathrm{x}}$ es la masa específica de la especie química o biológica $(\mathrm{X})$, las tasas de entrada y salida del flujo de masa se definen po $\dot{\mathrm{m}}_{\mathrm{x}, \text { in } \mathrm{y}} \dot{\mathrm{m}}_{\mathrm{X}, \text { out }}$, respectivamente $\mathrm{y}$ $\dot{r}$ es la tasa de generación de masa neta para cada especie [13]. Asimismo, las características del sustrato de entrada se dividen en concentraciones solubles (S), concentraciones particuladas (X) y en parámetros operacionales (flujo volumétrico y temperatura de operación). Estas variables se agrupan en una matriz Petersen, donde interactúan con 19 procesos bioquímicos mediante tasas cinéticas, parámetros estequiométricos y físicos que pueden consultarse en el reporte original del modelo, [9].

Por otro lado, para un buen funcionamiento del modelo varios autores recomiendan cambiar algunos valores en los parámetros estequiométricos, bioquímicos y fisicoquímicos del modelo dependiendo del tipo de residuo que se vaya a utilizar, [14]. De igual forma, se debe realizar una buena caracterización de los residuos de entrada y del sustrato en estado estacionario dentro del reactor para obtener resultados confiables sobre la producción de biogás, [15]. Una descripción más completa de las variables y el funcionamiento del ADM 1 ha sido presentada por los autores y se puede encontrar en, [7].

\section{Modelo Rowen para turbina de gas}

El modelo de Rowen representa una turbina de un solo eje sin recuperación de calor y su implementación en MATLAB/Simulink $®$ está compuesto por cuatro procesos principales: el control de velocidad, el sistema de combustión, el sistema de compresor - turbina y el control de temperatura. Un esquema de este modelo se muestra en la Figura 2.

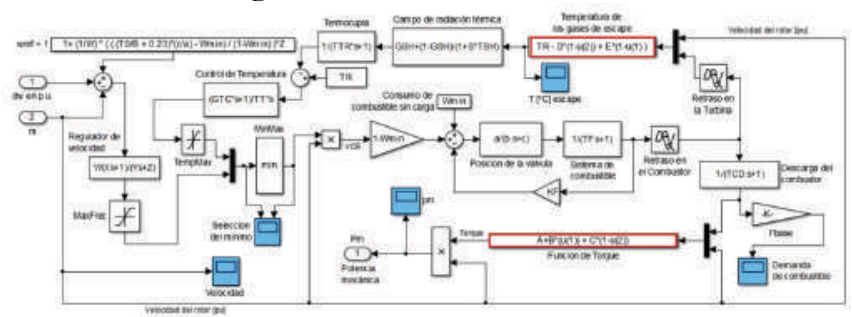

Figura 2 . Modelo de Rowen para una micro-turbina en MATLAB/Simulink $\mathbb{R}:[11],[16]$.

Estos bloques tienen en cuenta el torque generado, los gases de escape, la eficiencia de la combustión, el consumo de combustible y la potencia mecánica, [16]. Por su parte, los bloques de control mantienen a la TG dentro de sus límites de funcionamiento. Todas las magnitudes físicas están expresadas en por unidad (p.u) excepto las temperaturas que se encuentran en grados centígrados $\left({ }^{\circ} \mathrm{C}\right)$. Los parámetros $\mathrm{A}, \mathrm{B}, \mathrm{C}, \mathrm{D}$ y E que son usados en los bloques, los cuales representan el torque y la temperatura de los gases de escape (resaltados en la 
Figura 2 ), pueden ser calculados mediante la metodología propuesta en [16].

\section{Metodología}

\subsection{Estimación de la producción de biogás aplicando el ADM1}

Con el fin de brindar soluciones energéticas sostenibles en las ZNI se buscó un posible escenario local que ofrecieran buenas condiciones para la implementación de un SGEB. Los criterios de selección fueron enfocados en una alta producción de residuos provenientes de la industria bovina y una alta demanda eléctrica insatisfecha. La información de producción pecuaria del subsector bovino fue tomada del Censo Nacional Agropecuario [17] y la cantidad de usuarios locales sin cobertura eléctrica fue consultada en la base de datos del centro nacional de monitoreo $(\mathrm{CNM}),[2]$.

Con lo anterior, se logró identificar que el municipio ZNI con mayor producción de biomasa residual bovina en Colombia es San Vicente del Caguán, el cual cuenta con 612573 cabezas de ganado que producen alrededor de $6620000 \mathrm{~kg}$ de estiércol por día. Esta cantidad de residuo fue obtenida al multiplicar el número de animales por la tasa de producción de estiércol siguiendo el procedimiento expuesto en, [18].

Para el aprovechamiento de esta biomasa se supondrá un esquema de recolección centralizado, el cual consiste en la reunión de varios productores que concentran grandes cantidades de materia orgánica en un lugar específico para tratarla en una sola planta. Este esquema permite mayores tasas de producción de biogás, un aumento en la eficiencia, una reducción en los costos marginales de producción y una disminución en los errores de operación de la planta,[19]. No obstante, recolectar el 100\% del estiércol producido es muy difícil y costoso, por lo que algunos autores recomiendan un factor de recolección igual al 12,5\% [18], el cual hace referencia a la cantidad de biomasa residual que realmente está disponible. Para este estudio se supondrá que solo el $1 \%$ de toda la biomasa residual pecuaria de San Vicente del Caguán es recolectada, esto equivale a tener una producción de estiércol de $66204 \mathrm{~kg} /$ día.

Para calcular el caudal de entrada (qin) es necesario tener en cuenta que en reactores de agitación continua (CSTR) el estiércol es mezclado con agua generalmente èn una relación de 1:1,[20]. Además, siguiendo la recomendación de [21] se supone que el estiércol esta diluido, por lo que su densidad es similar a la del agua $(1000 \mathrm{~kg} / \mathrm{m} 3)$. Bajo estas suposiciones $\mathrm{qin}=132,4$ [m3/día]. Por otro lado, para determinar el tamaño del reactor se aplicaron las ecuaciones (2) a (5).

$$
\begin{gathered}
\text { TRH } \approx-51,227 * \ln T+206,72[\text { días }] \\
V_{\text {liq }}=T R H * q_{\text {in }}\left[\mathrm{m}^{3}\right] \\
V_{\text {total }}=V_{\text {liq }} / 0,75\left[\mathrm{~m}^{3}\right] \\
V_{\text {gas }}=V_{\text {total }}-V_{\text {liq }}\left[\mathrm{m}^{3}\right]
\end{gathered}
$$

Donde, $V_{l i q}$ es el volumen donde se almacenará el sustrato, TRH es el tiempo de retención hidráulica del mismo (24,6 días), $\mathrm{T}$ es la temperatura del reactor (generalmente $35^{\circ} \mathrm{C}$ ) y $V_{\text {gás }}$ es el volumen donde se acumula en primera instancia el biogás producido . Con esto se logró determinar para este estudio que $v_{\text {lia }} V_{\text {gás }} S o n$ $3253 \mathrm{~m} 3$ y $1078 \mathrm{~m} 3$, respectivamente.

El ADM1 es implementado en MATLAB/Simulink ${ }^{\circledR}$ de acuerdo a [24] . Las variables de funcionamiento del ADM1 están divididas en tres grupos: características del residuo de entrada, características de la materia orgánica dentro del biodigestor y un conjunto de parámetros estequiométricos, bioquímicos, fisicoquímicos y físicos. La caracterización del residuo de entrada fue tomada de [25]. Mientras que las condiciones iniciales y los parámetros fueron tomados de $[24],[25]$.

\subsubsection{Caracterización de la demanda eléctrica de la zona seleccionada}

Para facilitar la caracterización de la demanda se decidió tomar la localidad de San Vicente del Caguán que cuente con el mayor número de usuarios y con cero horas de cobertura. Bajo estos criterios, la zona selecciona fue San Juan de Caquetania con 234 usuarios. Sin embargo, la falta de información sobre las necesidades eléctricas de la población hace necesario utilizar una localidad cercana con condiciones similares para realizar una estimación de la demanda eléctrica.

Para cumplir con este requerimiento se usó la curva de carga promedio diaria de Solano de junio de 2017 que es la localidad con telemetría más cercana. Primero, se dividió la curva en el número de usuarios de solano (911) para obtener una relación de Potencia/usuarios y posteriormente se multiplico por el número de usuarios 
de San Juan de Caquetania (234) obteniendo la curva que se muestra en la Figura 3. En esta figura se observa la demanda mínima $(17,47 \mathrm{~kW})$, promedio $(29,97 \mathrm{~kW})$ y máxima de la localidad (46,23 kW). Adicionalmente, se puede calcular la energía diaria $(719,2 \mathrm{kWh})$ usando la demanda promedio multiplicada por 24 horas.

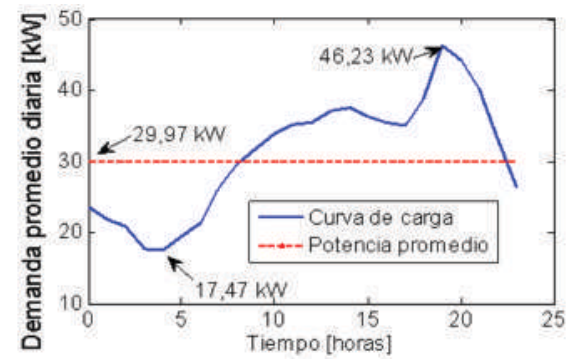

Figura 3 . Curva de carga aproximada para la localidad de San Juan de Caquetania. Fuente: elaboración propia

4.2. Esquema de generación de energía eléctrica a partir de biogás

Basándose en la potencia máxima mostrada en la Figura 3 se dimensionó el sistema de generación con una microturbina de $65 \mathrm{~kW}$ (referencia C65). Este valor se definió teniendo en cuenta que era el valor comercialmente disponible más cercano a la potencia máxima de la localidad. Estas turbinas están diseñadas para operar con biogás que no contenga ácido sulfhídrico y alcanzan eficiencias del 29\%. Por esta razón, se asume que el biogás producido es sometido a un proceso de purificación y luego es convertido a biometano para aumentar la densidad energética del biocombustible, [26].

En este arreglo, la turbina esta acoplada a un generador sincrónico que produce electricidad a $1600 \mathrm{~Hz}$. Debido a su alta velocidad de rotación (96000 rpm), es necesario adicionar una etapa de acondicionamiento de la señal donde se rectifique y luego invierta la tensión a $60 \mathrm{~Hz}$, [27]. Los parámetros operacionales de la microturbina C65 pueden verse en la tabla 1 y el esquema de generación implementado en MATLAB/Simulink $®$ se muestra en la figura 4.

\begin{tabular}{|l|l|l|}
\hline \multicolumn{1}{|c|}{ Parámetro } & Unidades & Valor \\
\hline Potencia nominal & $\mathrm{kW}$ & 65 \\
\hline Velocidad nominal & $\mathrm{rpm}$ & 96000 \\
\hline Relación de presión & - & 4,5 \\
\hline Eficiencia eléctrica & $\%$ & 29 \\
\hline Voltaje de salida (AC) & $\mathrm{V}$ & $400-$ \\
& & 480 \\
\hline
\end{tabular}

\begin{tabular}{|c|c|c|}
\hline Frecuencia & $\mathrm{Hz}$ & 60 \\
\hline Gases de escape & $\mathrm{Kg} / \mathrm{s}$ & 0,51 \\
\hline $\begin{array}{l}\text { Temperatura de los } \\
\text { gases de escape }\end{array}$ & ${ }^{\circ} \mathrm{C}$ & 311 \\
\hline $\begin{array}{l}\text { Consumo especifico } \\
\text { de combustible }\end{array}$ & MJ/kWh & 12,4 \\
\hline
\end{tabular}

Tabla 1. Parámetros operacionales de la microturbina C65 [28]. Nota: características obtenidas a condiciones estándar.

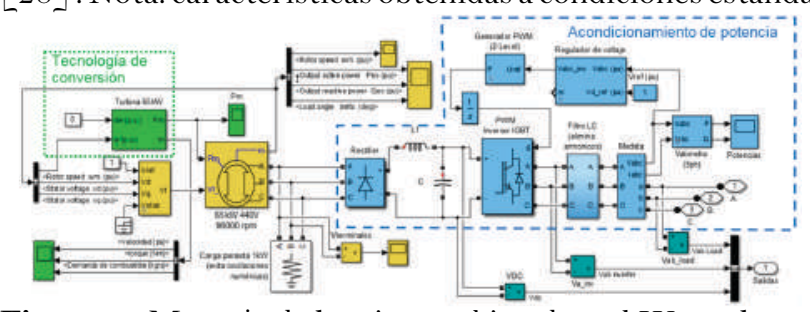

Figura 4. Montaje de la microturbina de $65 \mathrm{~kW}$ con la etapa de acondicionamiento de potencia. Fuente: elaboración propia.

Para el cálculo de los parámetros A, B, C, D y E asociados directamente a las funciones de torque y temperatura del modelo de Rowen se usó la metodología propuesta por [16]. Los valores restantes del modelo fueron obtenidos de estudios similares [27],[29] y los principales datos se presentan en la Tabla 2.

\begin{tabular}{|c|c|c|c|}
\hline Parámetros & Símbolo & Valor & Unidad \\
\hline $\begin{array}{l}\text { Constante para el bloque } \\
\text { de torque de salida }\end{array}$ & A & $-0,2485$ & - \\
\hline $\begin{array}{l}\text { Constante para el bloque } \\
\text { de torque de salida }\end{array}$ & $\mathrm{B}$ & 1,2485 & - \\
\hline $\begin{array}{l}\text { Constante para el bloque } \\
\text { de torque de salida }\end{array}$ & $\mathrm{C}$ & 0,5 & - \\
\hline $\begin{array}{l}\text { Constante para el bloque } \\
\text { de Temperatura de escape }\end{array}$ & $\mathrm{D}$ & 45863 & ${ }^{\circ} \mathrm{C}$ \\
\hline $\begin{array}{l}\text { Constante para el bloque } \\
\text { de Temperatura de escape }\end{array}$ & $E$ & 185,6 & ${ }^{\circ} \mathrm{C}$ \\
\hline $\begin{array}{l}\text { Límite máximo de señal de } \\
\text { demanda de combustible }\end{array}$ & MAX & 1,5 & p.u. \\
\hline $\begin{array}{l}\text { Límite mínimo de señal de } \\
\text { demanda de combustible }\end{array}$ & MIN & $-0,13$ & p.u. \\
\hline $\begin{array}{l}\text { Temperatura nominal de } \\
\text { escape }\end{array}$ & TR & 311 & ${ }^{\circ} \mathrm{C}$ \\
\hline Potencia base & $\mathrm{S}$ & 0,065 & MVA \\
\hline Eficiencia del compresor & $\eta_{\mathrm{c}}$ & 86 & $\%$ \\
\hline Eficiencia de la turbina & $\eta_{t}$ & 89 & $\%$ \\
\hline $\begin{array}{l}\text { Eficiencia de la } \\
\text { combustión }\end{array}$ & $\eta_{\text {comb }}$ & 80 & $\%$ \\
\hline
\end{tabular}

Tabla 2. Parámetros del modelo de Rowen para una turbina C65 [16]. 


\section{Resultados y análisis de resultados}

\subsection{Producción de biogás}

En esta etapa se simuló un reactor continuamente agitado con un caudal de entrada constante de 132,4 m /dia de estiércol bovino. En la a se puede observar la curva de producción de biogás, $\mathrm{CH} 4$ y $\mathrm{CO} 2$ en unidades de volumen. Para este caso, la composición del biogás fue aproximadamente de $67 \%$ de $\mathrm{CH} 4$ y $27 \%$ de $\mathrm{CO} 2$. Por su parte, la b muestra el $\mathrm{CH} 4$ producido en unidades de masa. Esta cantidad representa al biogás luego de pasar por el proceso de biometanización donde el CH4 es separado del resto de componentes del biogás. Si el CH4 se almacena durante los 100 días de simulación se obtienen en total $76868 \mathrm{~kg}$ de $\mathrm{Ch} 4$.
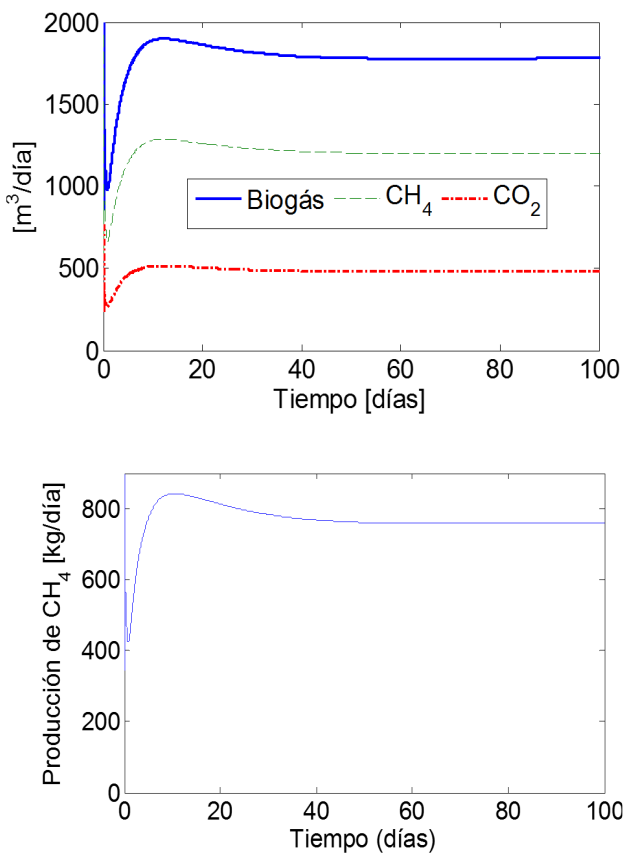

Figura 5 . Producción de biogás en unidades de (a) volumen y (b) $\mathrm{CH} 4$ en unidades de masa. Fuente: elaboración propia.

\subsection{Producción de electricidad}

El módulo de conversión biogás electricidad basado en una microturbina C65 es conectado a tres cargas que ingresan a la red de manera secuencial para representar la demanda mínima $(17,47 \mathrm{~kW})$, promedio $(29,97 \mathrm{~kW})$ y máxima $(46,23 \mathrm{~kW})$ de San juan de Caquetania (Ver figura 6a). En la figura $6 \mathrm{~b}$ se puede observar el comportamiento de la potencia mecánica generada por la microturbina. Al inicio, solo se encuentra conectada la carga mínima, lo que hace disminuir la potencia mecánica hasta 0,35 p.u. Después, en $t=10$ s se adiciona la segunda carga para obtener la demanda promedio. Luego, en $t=20 \mathrm{~s}$ la tercera carga es agregada completando la demanda máxima. Por último, en $\mathrm{t}=30 \mathrm{~s}$ la carga promedio y la carga máxima son desconectadas. Este deslastre de carga hace que la turbina vuelva al mismo estado reportado en $\mathrm{t}=10 \mathrm{~s}$.

a)

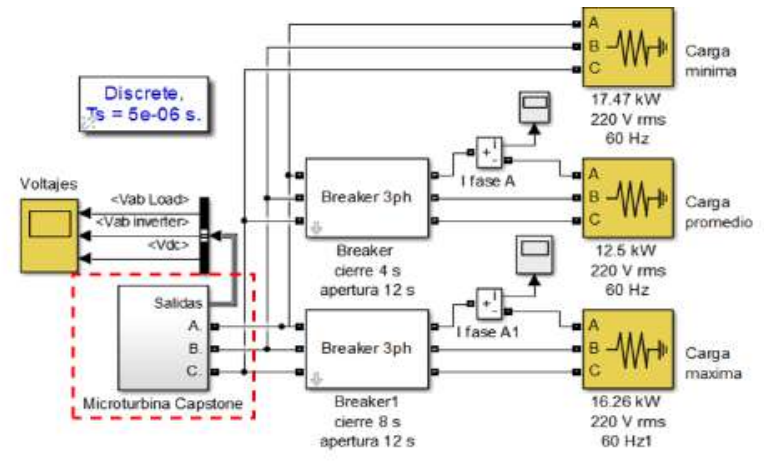

b)

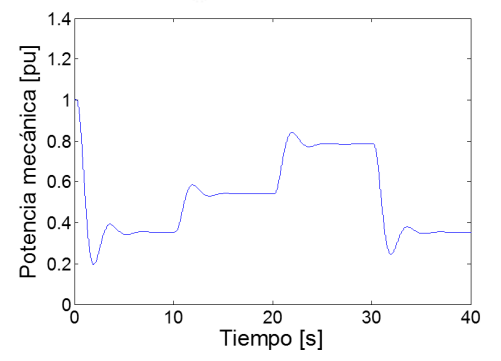

Figura 6 . (a) Esquema de conexión. (b)

Comportamiento de la potencia mecánica de la turbina ante variaciones en la carga. Fuente: elaboración propia.

Por otro lado, en la Figura 7a se observa que cuando existe poca carga el generador aumenta su velocidad y la disminuye a medida que el par de carga aumenta. Por su parte, la Figura 7b presenta la tensión DC antes de entrar al inversor, donde se evidencia una disminución de la tensión provocada por la conexión de las cargas resistivas. Al mismo tiempo, en la Figura 7c se pueden observar que la tension sobre la carga no ha cambiado. Esto se debe a que la modulacion realizada en el inversor es capaz de contrarestar estas pequeñas variaciones en la tensión. (a)

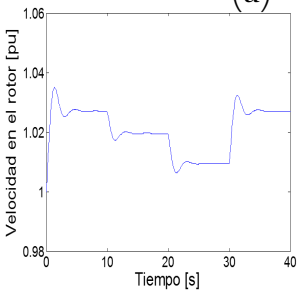

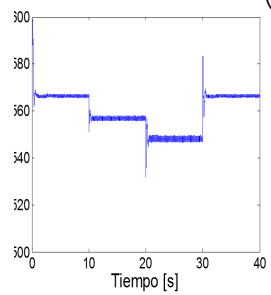

(b)

(C)

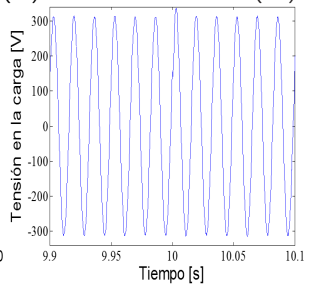

Figura 7 . Comportamiento de la (a) Velocidad. (b) Tensión DC. (c) Tensión en la carga, ante la variación secuencial de la carga. Fuente: elaboración propia. 
Para determinar el consumo promedio de combustible de la microturbina si opera todo el día con la carga promedio de San juan de Caquetania $(29,97 \mathrm{~kW})$, se realiza el montaje visto en la Figura 8 a. Los resultados pueden verse en la Figura $8 \mathrm{~b}$ donde el consumo de combustible se estabiliza después de 8 segundos en $0,002[\mathrm{~kg} / \mathrm{s}]$. Si se supone que se tiene almacenado todo el metano de la Figura $5 \mathrm{~b}(76868 \mathrm{~kg}$ de $\mathrm{CH} 4)$ es posible estimar el tiempo que la microturbina puede alimentar la carga promedio usando la ecuación(6).

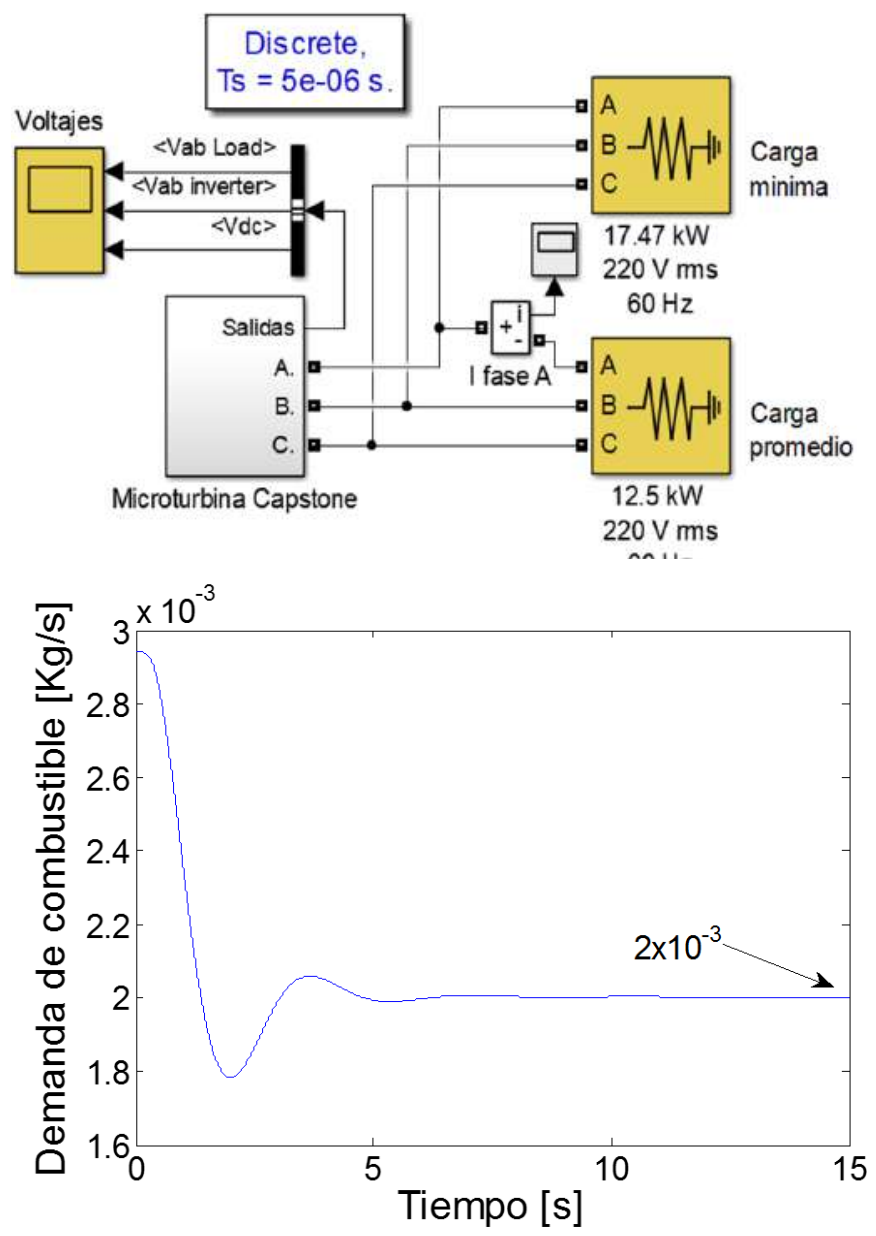

Figura 8. Sistema de conversión biogás-electricidad completo. Fuente: elaboración propia.

En este caso, el tiempo de funcionamiento es $76868 / 0,002=38434000$ s, lo que equivale a 10676 horas o 444 días. Esto quiere decir que la cantidad de $\mathrm{CH} 4$ almacenado durante los 100 días puede abastecer la carga promedio de San Juan de Caquetania $(29,97 \mathrm{~kW})$ durante poco más de 440 días.

\section{Conclusiones}

En las simulaciones se observa que cuando la carga es mínima, la turbina trabaja muy lejos de sus valores nominales (0,35 p.u). Para evitar que la turbina opere gran parte del día a carga parcial, la mejor solución puede ser instalar dos microturbinas de $30 \mathrm{~kW}$, lo que permitiría al sistema ser más eficiente y de paso más confiable. Otra alternativa podría ser complementar el sistema con otro tipo de fuentes que se encarguen de la demanda base.

Los resultados de simulación muestran que el uso de modelos matemáticos tanto para el proceso de DA como para el proceso de conversión biogás-electricidad facilitan la estimación del recurso energético disponible y el potencial de generación de electricidad que se puede obtener a partir del uso de diversas tecnologías de conversión. Todo esto sin incurrir en gastos previos, pruebas preliminares o la implementación de sistemas de conversión a partir de pruebas de ensayo y error.

Según los resultados obtenidos, si se logra recolectar diariamente el $1 \%$ del estiércol bovino generado en el municipio de San Vicente del Caguán y se almacena el $\mathrm{CH} 4$ producido durante 100 días, sería posible alimentar la demanda diaria de la localidad de San Juan de Caquetania durante un poco más de 440 días.

\section{Reconocimientos}

A la Universidad Distrital por su apoyo a través de la comisión de doctorado con el código de contrato $\mathrm{N}^{\circ}$ 000002-2016.

Referencias

[1] CNM-IPSE, "Centro Nacional de Monitoreo", 2017 . [En línea]. Disponible en: http://190.216.196.84/cnm/.

[2] CNM-IPSE, "Prestación de Servicio del CNM", 2017 . [En línea]. Disponible en: http://190.216.196.84/ps_cnm/.

[3] H. García, A. Corredor, L. Calderón y M. Gómez, "Análisis costo beneficio de energías renovables no convencionales en Colombia", FEDESARROLLO, Bogotá D. C, 2013.

[4] D. Deublein y A. Steinhauser, "Biogas from Waste and Renewable Resources", Deggendorf: WILEY-VCH, 2008. DOI: https://doi.org/10.1002/9783527621705. 
[5] K. C. Surendra, D. Takara, A. G. Hashimoto y S. K. Khanal, "Biogas as a sustainable energy source for developing countries: Opportunities and challenges", Renew. Sustain. Energy Rev., vol. 31,2014, pp. 846-859. DOI: https://doi.org/10.1016/j.rser.2013.12.015. s

[6] UPME, "Integración de las energías renovables no convencionales en Colombia", Bogotá: UPME, 2015.

[7] L. A. Morales, Á. D. Rodríguez y H. E. Rojas, "Assessment of the input substrate characteristics included in the anaerobic digestion model No.1", Revista Ingeniería, vol. 22, no. 2, 2017 , pp. 269-282. DOI: http://dx.doi.org/10.14483/udistrital.jour.re ving.2017.2.a07.

[8] A. Donoso-Bravo, J. Mailier, C. Martin, J. Rodríguez, C. Aceves-Lara y A. Vande Wouwer, "Model selection, identification and validation in anaerobic digestion: a review", Water Res., vol. 45, no. 17, 2011, pp. 5347-64. D O I :

https://doi.org/10.1016/j.watres.2011.08.059

[9] D. Batstone et al., "The IWA Anaerobic Digestion Model No 1 (ADM1)., Water Sci. Technol., vol. 45, no. 10, 2002, pp. 65-73.

[10] W. M. Budzianowski, "A review of potential innovations for production, conditioning and utilization of biogas with multiple-criteria assessment”, Renew. Sustain. Energy Rev., vol. 54,2016 , p p. $1148-1171$. DO I : https://doi.org/10.1016/j.rser.2015.10.054.

[11] W. Rowen, "Simplified mathematic representations of heavy-duty gas turbines", Eng. power, 1983, pp. 865-882.

[12] I. Ramírez, "Computation and implementation issues for biodiversity simulations with extended anaerobic digestion model no 1 (ADM1_N) in Matlab / Simulink", Rev. Invest. Univ. Quindío, vol. 24, no. 2, 2013, pp. 269-279.

[14] S. K. Khanal, Anaerobic Biotechnology for Bioenergy Production: Principles and Applications, 1st ed. Iowa, Estados Unidos: Wiley-Blackwell, 2009.

[14] M. Lübken, M. Wichern, M. Schlattmann, A. Gronauer y H. Horn, "Modelling the energy balance of an anaerobic digester fed with cattle manure and renewable energy crops", Water Res., vol. 41, no. 18, 2007, pp. 4085-96.

[15] U. Jeppsson, "Investigation of Anaerobic Digestion Alternatives for Henriksdal's
WWTP”, Lund, Sweden, 2007.

[16] M. R. Bank, B. Vahidi y W. Gawlik, "An educational guide to extract the parameters of heavy duty gas turbines model in dynamic studies based on operational data", IEEE Trans. Power Syst., vol. 24, no. 3, 2009, pp. $\begin{array}{lllllllll}136 & 6 & -1374 & \text { D O I : }\end{array}$ https://doi.org/10.1109/TPWRS.2009.2021 231.

[17] ICA, "Censo Pecuario Nacional 2016", Instituto Colombiano Agropecuario, 2016. [En línea]. D i s p o n i b l e e n : http://www.ica.gov.co/getdoc/9c3ad 7df-

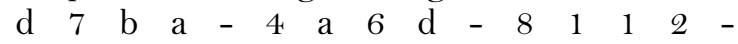
563caa9d8b17/Pecuaria.aspx.

[18] A. F. Ladino Tamayo y J. A. Martinez Rojas, "Metodología Para el Aprovechamiento Energético de Recursos de Biomasa Residual Pecuaria en la Autogeneración de electricidad: Casos de Estudio Briceño Boyacá y Cajicá Cundinamarca”, tesis, Universidad Distrital Francisco Jose de Caldas, Bogotá, Colombia, 2016.

[19] I. Angelidaki y L. Ellegaard, "Codigestion of manure and organic wastes in centralized biogas plants: status and future trends", Applied Biochemistry and Biotechnology, vol. 109, no. 1-3, 2003, pp. 95-105. DOI: https://doi.org/10.1385/ABAB:109:1-3:95.

[20] M. Garfí, J. Martí-Herrero, A. Garwood y I. Ferrer, "Household anaerobic digesters for biogas production in Latin America: A review", Renew. Sustain. Energy Rev., vol. 60, 2016, pp. $599-614$. D O I : https://doi.org/10.1016/j.rser.2016.01.071.

[21] UPME, "Guía para la implementación de sistemas de producción de biogás”, Bogota, Colombia, 2003.

[22] J. Martí-Herrero, "Biodigestores familiares: Guia de diseño y manual de instalación", La Paz: Cooperación Técnica Alemana GTZ, 2008.

[23] R. Botero y T. Preston, "Biodigestor de bajo costo para la producción de combustibles y fertilizante a partir de excretas", Cali: CIPAV, 1987.

[24] C. Rosen y U. Jeppsson, "Aspects on ADM1 Implementation within the BSM 2 Framework", Tech. Rep., 2008, pp. 1-37.

[25] A. Normak, J. Suurpere, K. Orupõld, E. Jõgi y E. Kokin, "Simulation of anaerobic digestion of cattle manure", Agron. Res., vol. 10, 2012, pp. 167-174. 
[26] W. M. Budzianowski, C. E. Wylock y P. A. Marciniak, "Power requirements of biogas upgrading by water scrubbing and biomethane compression: Comparative analysis of various plant configurations", Energy Convers. Manag., vol. 141, 2017, pp. 2-19. DOI: https://doi.org/10.1016/j.enconman.2016.03. O18

[27] D. N. Gaonkar y R. N. Patel, "Modeling and Simulation of Microturbine Based Distributed Generation System", IEEE Power India Conference, 2006, pp. 256-260. DOI: https://doi.org/10.1109/POWERI.2006.163 2521.

[28] M. A. Rosa do Nascimento et al., "Micro Gas Turbine Engine: A Review”, Prog. Gas Turbine Perform., 2013, pp. 107-141.

[29] S. R. Guda, C. Wang y M. H. Nehrir, "A simulink-based microturbine model for distributed generation studies", Proc. 37th Annu. North Am. Power Symp., 2005, pp. 269-274. DOI:

https://doi.org/10.1109/NAPS.2005.156053

7. 